\title{
Da dove vengono, cosa significano e come si usano sfiga, sfigato e (che) figo/fico
}

Michele Loporcaro

PUBBLICATO: 31 MARZO 2020

\section{Quesito:}

Un lettore pone un quesito mettendo insieme alcune parole apparentemente tutte connesse: "Mi chiedo perché la parola fico/figo indicante normalmente il frutto dell'albero omonimo (e le grazie femminili se il sostantivo è al femminile [...]) abbia assunto la connotazione gergal-giovanile di 'bello, ganzo, sorprendente'. Come ci si è arrivati? E come si è arrivati ad usare la medesima parola per descrivere un/a bello/a ragazzo/a? E per quanto riguarda la parola sfiga invece?". Su quest'ultimo termine chiedono spiegazioni anche una lettrice e un altro lettore che ipotizzano una possibile correlazione con il termine gergale fica e/o figa. Su quest'ultimo termine chiedono spiegazioni anche una lettrice ("Vorrei sapere come e da che cosa è nata la parola sfiga, se ha qualche correlazione con il termine gergale fica ofiga e se è una parola maschilista") e, con formulazione sintomaticamente diversa (che qui non riteniamo opportuno riportare per esteso), un altro lettore, il quale chiede se sia "iellato chi non dispone" liberamente del denotato del termine di cui sopra.

\section{Da dove vengono, cosa significano e come si usano sfiga, sfigato e (che) figo/fico}

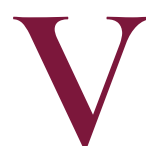

a premesso che nella lingua, e in particolare nell'etimologia, le cose a volte sono come appaiono, a volte no. Del primo tipo è il caso del nome dell'isola dell'Asinara, che deriva effettivamente dagli asinelli (bianchi) selvatici che la popolano, e non dal latino sinus 'sinuosità' nonostante le forme Sinnara e sim. delle carte rinascimentali, ricondotte a un *sinuaria, che costituiscono nobilitazione latineggiante secondaria rispetto all'Asenara della Carta Pisana del I275 (cfr. Carla Marcato, Asinara, in Giuliano Gasca Queirazza, Carla Marcato, Giovan Battista Pellegrini e Giulia Petracco Sicardi, Dizionario di toponomastica. Storia e significato dei nomi geografici italiani, Torino, UTET, I990, pp. 43-44). Se qui le cose stanno in effetti cosi come a tutta prima sembrano, d'altro canto l'Aspromonte, data l'originaria grecofonia dell'area in cui sorge, prenderà il nome più probabilmente dal biancore delle rocce (neogreco áspros 'bianco') che non dall'asperità del rilievo (lat. asper) con cui invece s'interpreta il nome nella percezione del parlante italiano medio (per le due ipotesi v. Giovan Battista Pellegrini, Toponomastica italiana, Milano, Hoepli, 20o8, p. 238). Similmente, benché la parola sfigato 'sfortunato, poveraccio, persona insignificante' esprima un concetto in certa misura agli antipodi di un (tipo) fico/figo, e nonostante i più fra i parlanti e i lessicografi li considerino connessi etimologicamente, $\mathrm{i}$ due aggettivi in realtà non condividono la stessa base e non hanno la medesima origine.

Palmare è quella di sfigato, aggettivo di forma participiale che, esattamente come il sinonimo sfortunato derivato da fortuna, è formato parasinteticamente (ossia, con l'applicazione simultanea di un prefisso e un suffisso: qui s-e -ato) dal sost. figa, variante settentrionale di fica 'pudendum muliebre'. Dall'aggettivo sarà stato tratto a sua volta il sostantivo sfiga 'sfortuna' (come l'aggettivo, in circolazione dagli anni Settanta), dato che meno plausibile sarebbe una formazione di quest'ultimo direttamente dall'altro sostantivo (per la derivazione di sfiga da sfigato si pronuncia il Vocabolario Treccani 2008, mentre per una derivazione in senso inverso optano il GRADIT e lo Zingarelli 20I7; infine, il GDLI deriva, ancor meno plausibilmente, sia sfiga sia sfigato direttamente da figa). 
Il procedimento di formazione presuppone ovviamente una visione maschilista per cui l'aver accesso al denotato è condizione fortunata, col che si risponde affermativamente al quesito posto dalla lettrice e dal secondo lettore. La risposta è però affermativa solo quanto all'origine prima, dato che, divenendo di larga circolazione, le parole sfiga e sfigato hanno perso di pregnanza scadendo al rango di semplici sinonimi più coloriti di sfortuna e sfortunato: se il GDLI le diceva ancora "del linguaggio volg[are]", gli altri vocabolari sopra citati le qualificano come del linguaggio giovanile (VocabolarioTreccani), fam(iliare) (GRADIT), colloq(uiale)/pop(opolare) (Zingarelli 20I7) registrando lo stingimento dell'originaria coloritura volgare. Le questioni che si pongono per l'origine e il significato di sfiga e sfigato sono dunque solo di dettaglio, come nel caso dell'Asinara.

Ben più controversa è invece la relazione con esse, e dunque l'origine, di (che) fico/figo!, di cui non è pacifico in realtà neppure il significato primario (e originario). Anche questa voce circola in italiano, originariamente nel linguaggio giovanile, sin dagli anni Settanta del Novecento: GRADIT dà come data di prima attestazione il I972, giustamente, sulla scorta di Cortelazzo e Cardinale (I989, p.I03), mentre nei supplementi retrodata al 1959 , erroneamente, come vedremo più in là. I dizionari la spiegano concordemente nel modo qui esemplificato con la voce dello Zingarelli 20r9:

fico (3) o (sett.) figo (2) [prob. da fica 1959] agg. e s. m. (pl. m. -chi; superl. fichissimo) - spec. nel gergo giovanile, che (o chi) incontra pienamente il gusto del momento, perché piacevole fisicamente, attraente, alla moda $\|$ fichetto, dim..

La voce è di irradiazione romana e almeno in origine, non è primariamente riferita alla sfera estetica, come invece dicono molti dizionari (v. anche il Devoto-Oli 20I4: "Alla moda, che riscuote o sollecita approvazione, compiacimento, complimenti"): un tipo fico è invece in primo luogo un 'tipo in gamba, che sa il fatto suo'; in questa direzione vanno le definizioni di altri dizionari, mettendo in secondo piano l'aspetto estetico: così il GRADIT: "che, chi piace per la sua bravura, la simpatia, la bellezza, l'eleganza e sim."). Questo significato primario, fra altri argomenti, e stato fatto valere da chi scrive per proporre una revisione dell'etimologia tradizionale che si legge nei dizionari italiani, etimologia che - come si vede nella voce Zingarelli ora riportata - presentafico/figo come un derivato del medesimo sostantivo femminile di cui sopra (Loporcaro I995; 1998; 2005).

Gli argomenti contro questa spiegazione vulgata sono da un lato di natura strutturale, dall'altro storici. Il piu forte tra i primi fa perno sul fatto che fico - pur passibile certo anche di uso sostantivato (è un fico) - è però un aggettivo, come correttamente indica il Vocabolario Treccani, mentre altri dizionari, come ora esemplificato con la voce Zingarelli (cosi anche Devoto-Oli 20I4, GRADIT, ecc.) indicano "agg. e s. m." cosi confondendo un po' le acque, e in modo non coerente dato che ovviamente non fanno lo stesso per aggettivi come bello, forte e sim. A ciò si aggiunge la coesistenza, a Roma, del sinonimo ficaccio, anch'esso aggettivo. Anche quest'ultimo conosce l'uso sostantivato (è un ficaccio), com'è normale per gli aggettivi. L'uno e l'altro formano però comparativo e superlativo, come aggettivi, appunto, e diversamente dai nomi: nel romanesco cui sono stato esposto negli anni Settanta si diceva una moto fichissima o, indifferentemente, ficaccissima.

Apparentemente, la relazione tra fico e ficaccio è la stessa che si ha ad es. tra fusto e fustaccio: ma qui, come per l'Aspromonte, l'apparenza inganna. Infatti, il suffisso -accio forma esclusivamente nomi a partire da altri nomi, mai aggettivi a partire da altri aggettivi, e ciò nonostante molte grammatiche indichino dei "derivati di base aggettivale" come avaraccio, bravaccio, caldaccio, maschiaccio ecc. Questi ultimi, infatti, sono formati apponendo il suffisso -accio non direttamente all'aggettivo bensi al suo omofono convertito in sostantivo: da (un gran) caldo si forma (un gran) caldaccio, mentre un giorno molto caldo non può diventare *un giorno molto caldaccio. Se dunque -accio forma esclusivamente 
sostantivi da sostantivi, e se ficaccio è invece, come fico, un aggettivo, ne discende necessariamente che il primo non può derivare dal secondo.

Quest'indicazione, evinta dal rapporto strutturale tra le due forme, è confermata dalla cronologia delle attestazioni, dato che ficaccio è attestato un secolo e mezzo prima difico nel senso prima indicato, in un sonetto belliano del 3r agosto i835: "Sapete? er fijjo de Monzú Bbojetto / Ha scuperto che un po' de corallina / È la vera e fficaccia mediscina / Pe gguarí sto fraggello bbenedetto" scrive infatti Giuseppe Gioachino Belli in uno dei sonetti della corona Er cóllera móribbus dedicati ai rimedi popolarmente ritenuti efficaci per un'epidemia di colera (cito dall'ed. a cura di Giorgio Vigolo, Milano, Mondadori, I952, n. 3016). Questo ficaccio è dunque una storpiatura popolare - certo non invenzione di Belli, che si fa anzi un punto d'onore di utilizzare solo parole e costrutti effettivamente ascoltati dalla viva voce della plebe romana - dell'aggettivo italiano efficace, storpiatura come se ne trovano $a b$ antiquo anche in altri dialetti italiani (in Loporcaro 2005, p. 348 se ne addita un esempio napoletano quattrocentesco). Da ficaccio, nel linguaggio giovanile della Roma degli anni Settanta, è stato poi formato l'aggettivo fico allo stesso modo in cui si sono formate varie altre parole, per sottrazione di suffisso: fascio, spago, spino per (e da) rispettivamente fascista (come in fascio, 'ndo te pijo te lascio, che si scandiva in rima baciata nei cortei), spaghetto (come in fámose du' spaghi), spinello ecc.

Siamo partiti col dire che i parlanti - e con loro molti lessicografi - considerano l'aggettivo fico/figo connesso al sostantivo fica/figa. Altri lo riconnettono a fico nome del frutto, da cui può derivare lo stesso sostantivo fica, di cui però è stata proposta autorevolmente (Cortelazzo 1970, pp. 80-8I) un'etimologia alternativa postulando una variante (*phếkē) del gr. thếke 'guaina', con uno scambio fra ph e th altrimenti attestato e con la stessa evoluzione semantica del lat. vagina 'guaina'.

Il richiamo al nome del frutto come origine del nostro aggettivo potrebbe esser motivato in base all'espressione romana er mejo fico der bigonzo: anche quest'etimologia pone problemi dal punto di vista morfologico - poiché non spiega la natura di aggettivi di fico/ficaccio - ma va comunque menzionata perché spiega invece la retrodatazione erronea sopra citata dell'aggettivo fico al I959, operata nei supplementi del GRADIT. Nel testo che motiva tale retrodatazione si legge: "A Zimmi, sei er mejo figo de la borgata!" (P. P. Pasolini, Una vita violenta, I959; v. D’Achille 20I2, p. 90 n. 28). Si tratta di una variazione sullespressione romana ora ricordata, in cui ricorre il sostantivo fico (frutto; con registrazione della lenizione intervocalica del romanesco). Er mejo fico der bigonzo significa "la migliore tra varie cose", come scrive Filippo Chiappini che registra la locuzione nel suo Vocabolario romanesco (v. a p. 46 e i26 ss. vv. fico e bigonzo 'bigoncia'). Quest'espressione romana preesiste, e di molto (Chiappini morì nel I905), all'insorgere dell'agg. fico, con cui si è incontrata secondariamente una volta nato quest'ultimo. È dunque evidente che il sostantivo (er mejo) figo usato da Pasolini nel I959 non può essere invocato a datare l'aggettivo fico/figo di cui qui parliamo.

Concludiamo riprendendo l'altra questione della modificazione in direzione estetica dell'accezione originaria che ha portato fico/figo da 'in gamba' a 'elegante' a 'piacevole, attraente'. Anche questo sviluppo semantico è dovuto ad un accostamento secondario o, come si dice tecnicamente, a una paretimologia. Per capire come ci si sia arrivati, si può partire di nuovo dalla voce citata dello Zingarelli, che registra fichetto sotto fico come un suo derivato. Cosi fa anche, più articolatamente, il Vocabolario Treccani:

fico $^{\mathrm{I}}$ (anche figo) agg. [...] Nel linguaggio giovanile, di persona abile, astuta, che si fa ammirare per qualche sua particolare capacità [...] • È usato anche al femm., e nei dim. fichétto, fichino (o fighétto, fighino), riferiti soprattutto, come sost., a ragazzo frivolo, che ostenta abbigliamento e atteggiamenti legati alla moda. 
Come qui giustamente si osserva, però, fichetto e fichino sono sostantivi: si dice un fichetto, non *un ragazzo fichetto. L'effetto di agrammaticalità è ancor più chiaro con l'altra formazione parallela fichino: impossibile dire *una festa fichina. Ora, diversamente dal suffisso -accio, i suffissi -etto e -ino si combinano sia con sostantivi (per es. bacetto, bacino da bacio) sia con aggettivi (per es. da stanco si forma stanchino e, meno frequentemente, stanchetto). Ma esattamente come accio e come tutti i suffissi alterativi, anche -etto e -ino non modificano la categoria lessicale della base: un nome resta un nome, un aggettivo resta un aggettivo. Se dunque fichetto è un nome, anche la sua base deve esserlo: non può quindi esser derivato dall'aggettivo fico, diversamente da quanto lascia intendere quell'"è usato anche [...] nei dim. fichétto, fichino". Serve invece un sostantivo.

Anche qui la prova strutturale ora addotta converge con quella storica: infatti sia l'aggettivo fico che il sostantivo fichetto circolano in italiano solo dagli anni Settanta del Novecento, ma il secondo, a differenza del primo, è parte di una schiera di varianti (fighetto, - $a$ e, con diversa suffissazione diminutiva, fichino) largamente attestate sin dall'Ottocento nei dialetti di tutta la Penisola come termini ingiuriosi riferiti a un uomo. Per es. (don) fichino è "nome che [...] si dà a giovane bellimbusto, damerino" secondo l'ottocentesco Vocabolario napoletano italiano di Raffaele Andreoli (Torino, Paravia, I887, p. 274), mentre fichetto come epiteto ingiurioso rivolto a uomo ricorre in vari sonetti belliani, ad es. nella serie di quarantatré epiteti del sonetto n. 2032 (ed. Vigolo I952). Questi sostantivi dialettali si hanno anche al nord: ad es. ferrarese fighin e fighèt, anche qui sin dall'Ottocento, "t. di scherno" col valore di 'bellimbusto' nel Vocabolario ferrarese-italiano di Luigi Ferri (Ferrara, Tipografia Sociale, I889, p. I45); e poi bolognese figàtt 'damerino, effeminato' (corrispondente formalmente a fichetto) e molti altri.

Il significato di questi sostantivi dialettali si spiega naturalmente a partire dal sostantivo femminile di base (fica/figa) - usato largamente in tutti i dialetti per formare epiteti ingiuriosi rivolti a uomo - con l'aggiunta del valore diminutivo: se ne ottiene, originariamente nei dialetti, uno pseudo-vezzeggiativo di scherno che fa leva sull'idea di effeminatezza. Una volta pero entrato in circolazione l'aggettivo fico con valore di apprezzamento, questo e i sostantivi in -ino e -etto si sono reciprocamente attratti e influenzati. Cosi, fichetto ha finito per esser sentito come riconducibile a fico (teste anche la modalità di registrazione nei dizionari italiani) e d'altro canto quest'ultimo ha virato in direzione della valutazione estetica, centrale ab origine (pur nella connotazione negativa) per i dialettali fichetto e fichino.

Insomma, alla domanda se gli aggettivi figo e sfigato siano parole connesse si deve rispondere con un distinguo: non lo sono etimologicamente, ma lo sono divenute nella coscienza dei parlanti.

\section{Nota bibliografica:}

- Filippo Chiappini, Vocabolario romanesco. Ed. postuma delle schede a cura di Bruno Migliorini, con aggiunte e postille di Ulderico Rolandi, Roma, Leonardo da Vinci, $1945^{2}$.

- Manlio Cortelazzo, L'influsso linguistico greco a Venezia, Bologna, Pàtron, I97o.

- Manlio Cortelazzo e Ugo Cardinale, Dizionario di parole nuove (1964-1987), Torino, Loescher, $1989^{2}$.

- Paolo D'Achille, Parole nuove e datate. Studi su neologismi, forestierismi, dialettismi, Firenze, Cesati, 2012.

- Michele Loporcaro, Un problema d'etimologia: sul che fico! del linguaggio giovanile, "Studi di lessicografia italiana" I3, I995, pp. 343-364. 
- Michele Loporcaro, L'avventura di un povero linguaiolo: ancora sull'etimologia di che fico, "Rassegna europea di letteratura italiana" II, I998, pp. III-II7.

- Michele Loporcaro, Morfologia ed etimologia: alcuni esempi italo-romanzi, in La formazione delle parole. Atti del XXXVII Congresso internazionale di studi della Società di Linguistica Italiana (SLI), L'Aquila, 25-27 settembre 2003, a cura di Maria Grossmann, Anna M. Thornton, Roma, Bulzoni, 2005, pp. 335-350.

\section{Cita come:}

Michele Loporcaro, Da dove vengono, cosa significano e come si usano sfiga, sfigato e (che)

figo/fico , "Italiano digitale", 2019, XII, 2020/1 (gennaio-marzo)

DOI: $10.35948 / 2532-9006 / 2020.3276$

Copyright 2019 Accademia della Crusca

Pubblicato con licenza creative commons CC BY-NC-ND 